\title{
Co- and counter-helicity interaction between two adjacent laboratory prominences
}

\author{
J. F. Hansen, ${ }^{\text {a) }}$ S. K. P. Tripathi, and P. M. Bellan \\ Applied Physics, Caltech, Pasadena, California 91125
}

(Received 21 October 2003; accepted 3 March 2004; published online 11 May 2004)

\begin{abstract}
The interaction between two side-by-side solar prominence-like plasmas has been studied using a four-electrode magnetized plasma source that can impose a wide variety of surface boundary conditions. When the source is arranged to create two prominences with the same helicity (co-helicity), it is observed that helicity transfer from one prominence to the other causes the receiving prominence to erupt sooner and faster than the transmitting prominence. When the source is arranged to create two prominences with opposite helicity (counter-helicity), it is observed that upon merging, prominences wrap around each other to form closely spaced, writhing turns of plasma. This is followed by appearance of a distinct bright region in the middle and order of magnitude higher emission of soft $\mathrm{x}$ rays. The four-electrode device has also been used to change the angle of the neutral line and so form more pronounced S-shapes. (C) 2004 American Institute of Physics. [DOI: 10.1063/1.1724831]
\end{abstract}

\section{INTRODUCTION}

Solar prominences are large, arched plasma structures protruding from the surface of the Sun. ${ }^{1}$ A prominence can remain stable for several days, only to become suddenly unstable and erupt in a few minutes. It has been postulated that instability occurs when the magnetic forces acting on a prominence are no longer in balance, particularly when the hoop-force is too large for the restoring forces. The hoopforce is simply the repelling force between two antiparallel currents, in this case the prominence current on opposite sides of the prominence. The restoring forces are the $\mathbf{J} \times \mathbf{B}$ force between this current and a large scale strapping field, ${ }^{2}$ and the magnetic field line tension along the prominence. An increase in the prominence current is equivalent to an increase in magnetic helicity in the prominence. The exact mechanism for helicity injection has not been ascertained but two distinct theories have been proposed, namely:

(1) There is a gradual build-up of magnetic energy and helicity in a single magnetic structure which then erupts producing a prominence eruption, coronal mass ejection, or flare depending on the size and shape of the structure.

(2) Two or more existing structures merge, combining their helicity to make the resulting structure unstable $e^{3-6}$ and eruptive.

Both theories are plausible and both situations might occur. The solar dynamo could act to inject sufficient helicity into a single structure to make the structure unstable or, on the other hand, unstable prominences could form when twisted ${ }^{7}$ magnetic flux tubes "bubble up" from the solar photosphere, ${ }^{8}$ and then merge with an already existing prominence adding their helicity to the existing prominence.

\footnotetext{
${ }^{a)}$ Present address: Lawrence Livermore National Laboratory, Livermore, California 94550-9234.
}

This sequential addition of several small flux tubes according to the second theory would eventually build up the prominence current and helicity to the point where instability occurs.

It is not difficult for two prominences to be sufficiently close to each other to interact and, in particular, the two prominences will attract each other if their axial currents are parallel. Interaction between two prominences of the same handedness (co-helicity) should be somewhat more common on the Sun than interaction between prominences of opposite handedness (counter-helicity). The reason for this is that the handedness of solar prominences has been shown to be preferentially of the same sign in each hemisphere-right handed (positive helicity) in the southern hemisphere of the Sun, and left handed (negative helicity) in the northern hemisphere. ${ }^{9-12}$ In a co-helicity interaction, the angles between reconnecting magnetic fields are much less than in the counter-helicity case, and because of this difference, cohelicity interaction is expected to release considerably less magnetic energy than counter-helicity interaction. The less frequent, but more energetic counter-helicity interaction could play a role in the origin of solar flares, ${ }^{13}$ although it should be noted that not all flares involve prominences.

This paper describes initial results from an experiment designed to explore the various possible interactions between two prominence-like plasmas. These interactions should be relevant to solar prominence formation, helicity build-up, and eruption. The results show that there exist distinct types of interactions depending on the relative handedness of the interacting prominences. The results also show that the nature of the prominence depends on the orientation of the neutral line and on the mass flux surface boundary condition.

\section{EXPERIMENTAL SETUP}

In order to investigate how two prominences interact, we constructed a four-electrode magnetized-plasma gun capable 
(a) Co-helicity (RR)



(b) Counter-helicity (LR)

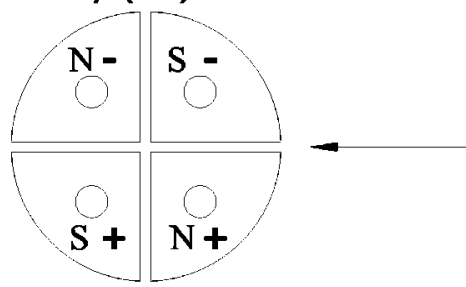

(c) Single prominence (R)



(d) Pronounced S-shape prominence (R)

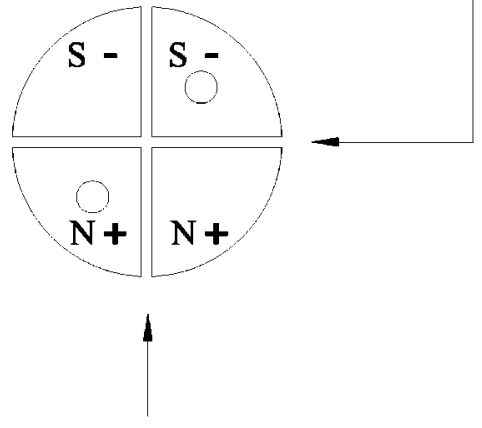

Parallel View

FIG. 1. Various operational configurations of the experiment, with the four electrodes shown from the top view (perpendicular view indicated from the right; parallel view from below). Anodes are marked with "+," cathodes with "-, ," electromagnet north poles with "N," electromagnet south poles with "S," and each gas injection point is marked with a circle. A co-helicity case with two right-handed (RR) prominences is shown in (a). A counterhelicity case is shown in (b). A single prominence case obtained by cutting off the gas in one pair of electrodes is shown in (c). Gas injection through a pair of diagonally placed electrodes is shown in (d), with this diagonal leading to prominences with pronounced S-shape.

of producing two side-by-side solar prominence-like plasmas; this gun is shown schematically in Fig. 1. The gun is mounted nearly flush with the end dome of a large $(2 \mathrm{~m}$ long, $1.4 \mathrm{~m}$ diam) vacuum chamber. The metal end dome simulates the flux-conserving surface of the Sun, and since all other walls are far away, the configuration approximates an infinite half-space. The plasma gun makes prominences in three steps. First, electromagnets (one under each of the four electrodes) create a pair of arched potential magnetic fields which protrude into the vacuum chamber. These bias fields are $0.3 \mathrm{~T}$ at the foot-points, which are $8.0 \mathrm{~cm}$ apart. Second, approximately $10^{-8} \mathrm{~kg}$ of hydrogen gas is injected through as many as four orifices (one at each foot-point of the arched potential field) into the region where the prominence will form. Third, a $59 \mu \mathrm{F}$ capacitor bank charged to $3-6 \mathrm{kV}$ is connected across the foot-points, causing the hydrogen gas cloud to break down and form a highly conducting plasma (resistance 0.02-0.1 $\Omega$ ). After breakdown, the discharging capacitor ramps up current through the plasma; this current reaches a maximum approximately $8 \mu$ s after breakdown. The peak current is proportional to the original capacitor bank voltage, and is approximately $40 \mathrm{kA}$ for a charging voltage of $3 \mathrm{kV}$.

The typical length scale of an actual solar prominence is $10^{7}-10^{8} \mathrm{~m}$ and the ratio of hydrodynamic to magnetic pressure, $\beta=2 \mu_{0} p / B^{2}$, is small, on the order of $10^{-3}-10^{-1}$, so that magnetic forces dominate hydrodynamic forces. The ratio of the diffusive time scale to the convective time scale (i.e., the Lundquist number, a measure of how well a magnetic field is frozen to the plasma) is large for a solar prominence, as high as $10^{14} \cdot{ }^{14,15}$ The laboratory prominence plasma has a measured density of $10^{19}-10^{20} \mathrm{~m}^{-3}$ and electron temperature $\sim 5 \mathrm{eV}$, corresponding to low $\beta$ $\left(10^{-4}-10^{-3}\right)$ and high Lundquist number $\left(10^{3}-10^{4}\right)$. The combination of low $\beta$, high Lundquist number, and experimental time larger than the Alfvén time $(\sim 0.05 \mu$ s using an estimated Alfvén velocity of $2 \times 10^{6} \mathrm{~m} / \mathrm{s}$ and assuming a typical length scale of $0.1 \mathrm{~m}$ ) but shorter than the resistive diffusive time $(\sim 300 \mu \mathrm{s})$ produces a reasonable simulation of the solar parameter regime. ${ }^{1,16}$

The four-electrode plasma gun permits a multitude of different operating conditions because the polarity of each of the four bias field coils can be set independently and because the gas can be selectively injected through any combination of bias field foot-points. If the bias field for a lab prominence is oriented such that this field is parallel to the prominence current, then a right-handed (positive helicity) prominence is created whereas if the bias field is anti-parallel to the prominence current, a left-handed (negative helicity) prominence is created. The situation where two interacting prominences have the same helicity (either both right-handed or both lefthanded) is called co-helicity and the situation where they have opposite helicity is called counter-helicity. The imposed boundary conditions on the four-electrode plasma gun for the co- and counter-helicity cases are shown in Figs. 1(a) and 1(b), respectively. In normal operation, gas is injected through all four orifices resulting in the creation of two neighboring prominences, but the four-electrode gun can also be configured to create a single prominence similar to that produced in earlier experiments ${ }^{2}$ by cutting off the gas flow to one pair of electrodes as shown in Fig. 1(c) so that the Paschen condition for gas breakdown is not satisfied and a second prominence does not form. Yet another boundary condition can be obtained by cutting off the gas flow for two diagonally opposite electrodes as shown in Fig. 1(d). This forces the prominence to form and evolve in a background potential field that is at a $45^{\circ}$ angle to a line between the prominence foot-points (in solar terminology this arrangement is called having a neutral line at $45^{\circ}$ ). 


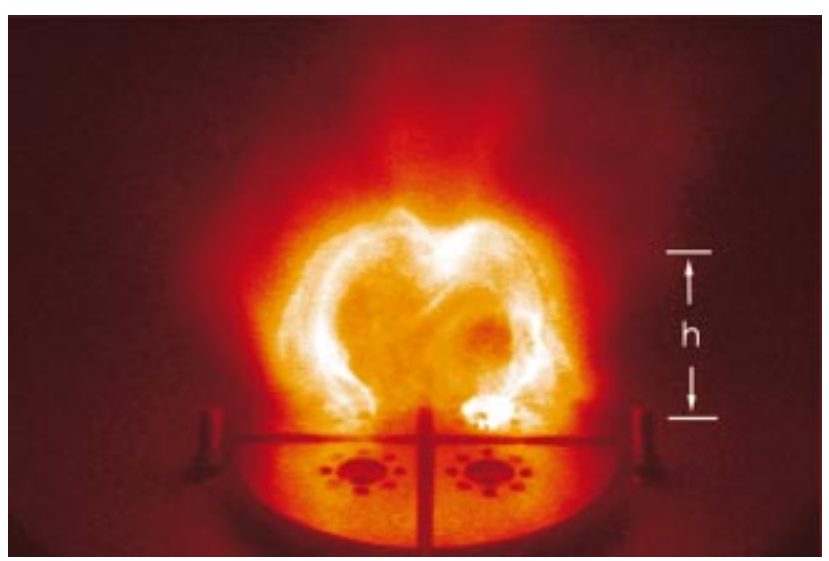

FIG. 2. (Color) Example of a single prominence (created by cutting off the gas flow through the two electrodes in the foreground). The distance $h$ is a measure of the plasma expansion (see Fig. 8).

The main diagnostics for these experiments consisted of (i) a single frame, gated, intensified, cooled charge coupled device (CCD) still camera having a 10 ns shutter speed (Manufacturer: Princeton Instruments, Model: ICCD-576), (ii) a 16-frame, gated, intensified, CCD framing camera (Manufacturer: DRS Technologies, Model: Imacon 200), and (iii) a high speed photodiode array sensitive to visible/ extended ultraviolet/soft x-ray (V/EUV/SXR) radiation. The diagnostics imaged and measured the prominences from three orthogonal directions (see Fig. 1): parallel, camera in the plane of prominence formation and pointing along the line between foot-points; perpendicular, camera pointing normal toward the plane of prominence formation; top, camera pointing toward the base plane representing the solar surface.

The still camera was used to take visual light snap-shots of the prominences at prescribed times during their evolution. These visual snapshots are highly reproducible and enable us to record the evolution of plasma structures with temporal resolution as small as $0.1 \mu \mathrm{s}$. An example image is shown in Fig. 2. Application of various filters to the camera has shown that the visual light plasma emission is mainly $\mathrm{H}_{\alpha}$. The framing camera provided better information on evolution than could be obtained from still camera photos.

The V/EUV/SXR photodiode array was constructed using three International Radiation Detector Corporation AXUV-HS5 diodes and was located on a movable stalk inside the vacuum chamber approximately $60 \mathrm{~cm}$ from the prominences with a top view. These diodes have subnanosecond risetimes, are sensitive to electromagnetic radiation from visible light to soft $\mathrm{x}$ rays, and have a quantum efficiency which increases with increasing photon energies (approximately $10 \%$ per eV). An incoming photon with an energy of $10 \mathrm{eV}$ will produce one electron that can be detected by an external circuit connected to the diode. To confine the sensitivity to a certain spectral range, diodes can be covered with thin metal foils. The V/EUV/SXR probe had one diode with no foil, one diode with a $200 \mathrm{~nm}$ aluminum foil which transmits EUV radiation in the wavelength range $20-80 \mathrm{~nm}$ (equivalent to photon energies of $15-62 \mathrm{eV}$ ), and one diode with a $50 \mathrm{~nm}$ titanium foil which transmits soft $\mathrm{x}$ rays with wavelengths $<15 \mathrm{~nm}(>83 \mathrm{eV})$. The probe was arranged so that the diodes were located behind a set of neodymiumalloy permanent magnets which had the function of warding off charged particles. The magnetic field strength in the spacing between magnets was $\geqslant 0.4 \mathrm{~T}$ which is sufficient to deflect incoming ions and electrons (an ion with a kinetic energy of $100 \mathrm{eV}$ would have an ion cyclotron radius of $\sim 4 \mathrm{~mm}$ and electrons would have even smaller cyclotron radii). Furthermore, a grid of nickel wires with a $127 \mu \mathrm{m}$ spacing (or 200 wires per linear inch) was placed between the magnets and the diodes. The diodes, bias grid, and magnets were enclosed in a metal probe head that allows photon entry to the diodes only through narrow channels passing the magnets and then the bias grid. In order to improve diode risetimes a reverse bias voltage of $31 \mathrm{~V}$ was applied to the diodes using a Picosecond Pulse Labs bias tee. To ensure maximum shielding from the noisy electrical environment, semi-rigid coaxial cable was used to connect to the diodes through Ceramaseal hermetically sealed SMA connectors welded onto a 2.75 in. Conflat flange. An SIS3300 digitizer with 12 bit resolution and $100 \mathrm{MHz}$ sampling rate was used to acquire the data. Fast response of the $\mathrm{x}$-ray diodes was ensured by the matched bias tee and also by terminating the co-axial cable with its characteristic $50 \Omega$ impedance at the digitizer input. The local ground of the diode array was isolated from the vacuum chamber ground to prevent ground loops and the entire diode array system was enclosed by an aluminum shroud at machine ground and insulated from the diode array ground. No variation in diode signal was observed when the grid was biased up to $\pm 100 \mathrm{~V}$, verifying that the magnets successfully diverted incoming ions and electrons from the diodes. These electrical shielding, particle diversion, and fast risetime measures were found to be necessary based on experience gained from using slow, poorly shielded photodiodes which had inadequate temporal response and which produced signals that were obviously influenced by charged particles.

\section{RESULTS}

For the results presented here, the charging voltage on the discharge capacitor is $6 \mathrm{kV}$, unless otherwise stated.

\section{A. Single prominence}

By careful comparison of images of the prominence evolution obtained using the three orthogonal camera directions, an understanding of the three-dimensional nature of the evolution was developed. The evolution of a single prominence [with boundary conditions as in Fig. 1(c)] has been previously reported. ${ }^{2}$ The plasma is initially shaped like a smooth, arched current channel which follows the applied potential magnetic field, similar to coronal field lines on the Sun. At these early times, the plasma looks like half of a torus, and it is useful to define the direction from one foot-point to the other-along the arching plasma-as the "toroidal" direction. As the toroidal current increases, the minor radius of the plasma decreases due to the pinch effect. At the same time, but on a finer spatial scale, the current channel decomposes into filaments. From a top view, the plasma looks S-shaped or reverse-S shaped. With its filamentary structure and over- 
TABLE I. Emission of $<15 \mathrm{~nm} x$ rays as measured by the diode with a $50 \mathrm{~nm}$ titanium foil for two prominences, in either co- or counter-helicity configuration, as well as for single prominences, as a function of charging voltage on the main discharge capacitor. Emission is peak diode voltage measured across a $50 \mathrm{ohm}$ resistor. The errors are standard deviations from average values, calculated from 10 to 15 plasma shots for a given condition.

\begin{tabular}{cccc}
\hline \hline $\begin{array}{c}\text { Main capacitor } \\
\text { charging voltage }(\mathrm{kV})\end{array}$ & $\begin{array}{c}\text { Single prominence } \\
\text { x-ray emission }(\mathrm{mV})\end{array}$ & $\begin{array}{c}\text { Co-helicity } \\
\text { x-ray emission }(\mathrm{mV})\end{array}$ & $\begin{array}{c}\text { Counter-helicity } \\
\text { x-ray emission }(\mathrm{mV})\end{array}$ \\
\hline 3.00 & $0.6 \pm 0.3$ & $0.8 \pm 0.6$ & $0.5 \pm 0.2$ \\
4.00 & $1.3 \pm 0.4$ & $1.1 \pm 0.6$ & $1.1 \pm 0.2$ \\
5.00 & $4.2 \pm 2.1$ & $2.3 \pm 1.5$ & $16.8 \pm 15.4$ \\
6.00 & $7.8 \pm 5.7$ & $3.6 \pm 1.6$ & $176.3 \pm 100.6$ \\
\hline \hline
\end{tabular}

all S-shape, the plasma now looks much like a prominence on the Sun. Magnetic hoop forces cause the major radius to expand. ${ }^{2}$ Eventually, the superposition of the self-magnetic field due to the current onto the original potential field causes the prominence to develop a helical shape. From a perpendicular view as shown in Fig. 2, the helical shape gives the illusion of the prominence having a sharp, cusp-like, central dip halfway between the foot-points, but this apparent dip is merely a projection of the helix, which can be thought of as writhing around the surface of a fictitious toroidal envelope. ${ }^{2}$ The cusp-like shape is visible also from a top view as the earlier, topologically simpler S-shape evolves into the helix. $\mathrm{X}$-ray output, as measured with the diode probe system, increases with increasing plasma current; the $\mathrm{x}$-ray emission tracks the current profile in time, and its magnitude depends on the initial capacitor bank charging voltage (see Table I).

\section{B. Co-helicity merging of two prominences}

When two prominences form and evolve side by side, they attract each other if their toroidal currents are parallel, since parallel currents attract. In the co-helicity case (see Fig. 3 ), it is observed that this interaction causes one prominence to expand faster than the other. In the figures, indicated times for frames during evolution of the plasma are measured from when the main discharge capacitor load is triggered. This unequal expansion is visible for all viewing angles (parallel, perpendicular, top). This is an important observation of a phenomena that could occur on the Sun. Which of the two prominences expands faster depends on whether the prominences are both right-handed or both left-handed. In the top view, with the cathode oriented above the anode, the prominence to the right expands faster when both prominences are right-handed, whereas the prominence on the left expands faster when both prominences are left-handed.

From the perpendicular and top views in the co-helicity case, it appears that the leading parts (meaning the parts furthest from the electrodes) of the two prominences merge, at least at the spatial scale resolved by the cameras. This merging is less apparent in the parallel view. The x-ray emission in the co-helicity case, for a given capacitor bank charging voltage, was of similar order to that of a single prominence case (see Table I).

\section{Counter-helicity merging of two prominences}

The evolution of two prominences in the counter-helicity configuration is shown in Fig. 4. In this configuration, radial streaks of plasma are seen emanating from the toroidal plasma arches during the initial stages of prominence evolution (between approximately $3.0 \mu \mathrm{s}$ to $4.5 \mu \mathrm{s}$ of the discharge). The co-helicity configuration lacks radial streaks (4.0 $\mu$ s frame in Fig. 3), while in the counter-helicity configuration plasma ejection along radial streaks is visible close to the anodes (4.0 $\mu$ s frame in Fig. 4).

Between $4.5 \mu \mathrm{s}$ and $5.5 \mu \mathrm{s}$, the initially formed toroidal plasma arches wrap around each other and merge to form closely spaced, writhing loops of plasma (see Fig. 4). The plasma structure at this stage has a strong dependence on the discharge voltage as seen in Fig. 5. At higher discharge voltage the number of loops increases, which suggests that the annihilation of the toroidal magnetic field is more effective at higher discharge currents. Additional images (not shown) show that radius of the loops increases with the discharge voltage, whereas the interloop spacing decreases. These features are not very clear in still frames but could be clearly seen in movies generated by the frames. These writhing turns disappear after $5.5 \mu$ s and a much brighter, twisted plasma arch is formed between $5.5 \mu$ s to $6.25 \mu$ s (Fig. 4).

The most significant observation in the counter-helicity merging is the formation of an intense bright region, after approximately $6.5 \mu \mathrm{s}$, halfway between the foot-points along the merged plasma column. The appearance of this bright region coincides with a sharp rise in the x-ray signal, which becomes more than an order of magnitude higher than its value for the co-helicity configuration (see Table I). The time dependence of the discharge voltage, discharge current, and $\mathrm{x}$ rays from single prominence, co-helicity merging, and counter-helicity merging configurations are shown in Fig. 6. Note that the sharp rise in the $\mathrm{x}$-ray emission for the counterhelicity configuration precedes the discharge current maximum, whereas the current and x-ray emission maxima coincide for both the single prominence and co-helicity configurations. When viewed from the perpendicular direction, the bright region appears spherical. We also find smooth arc segments attached to either side of this bright region, when viewed from the perpendicular direction as seen at 8.0 $\mu \mathrm{s}$ in Fig. 4. With an increase in discharge voltage, the bright 


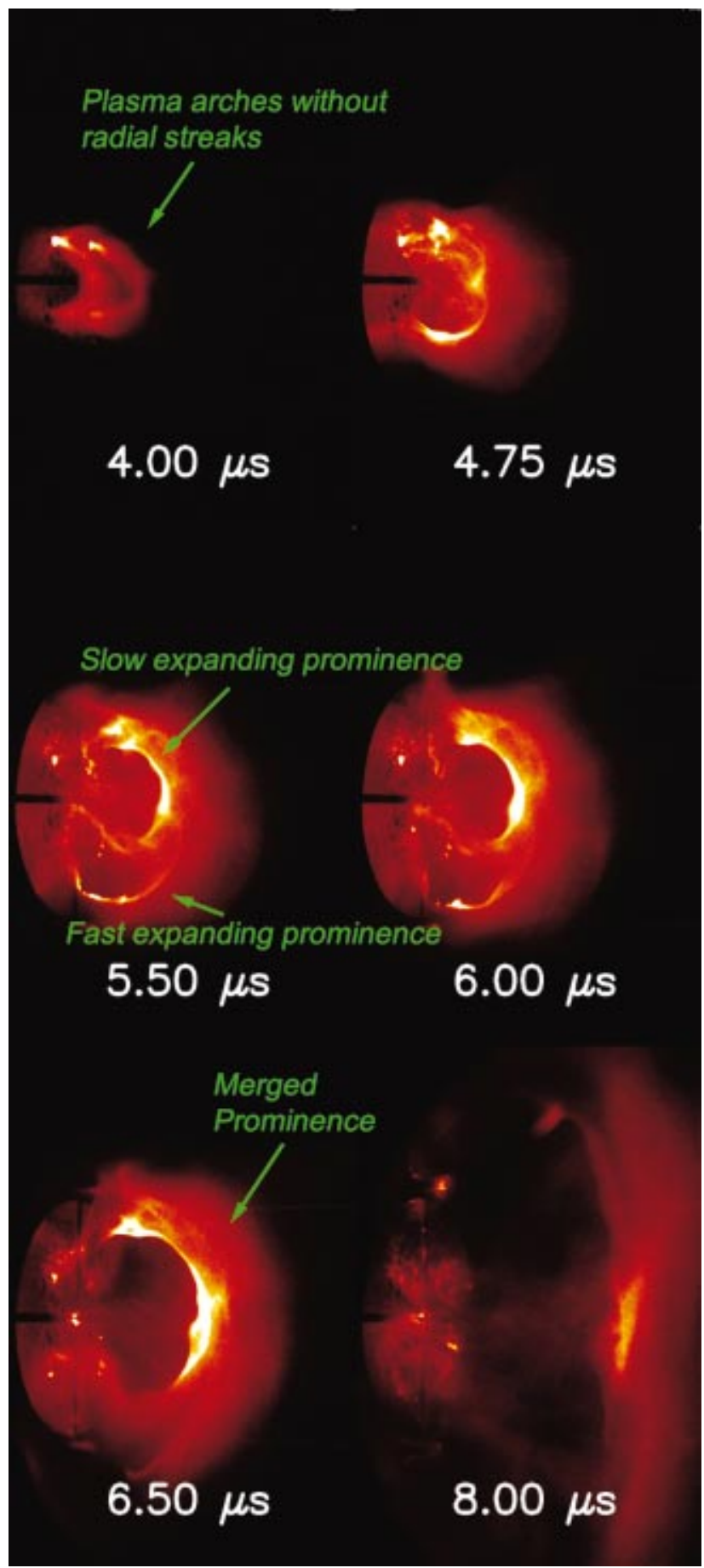

FIG. 3. (Color) Evolution of two prominences in the co-helicity case (perpendicular view). Indicated times are measured from when the trigger pulse is applied to the discharge capacitor bank (at $t=0$ in Fig. 6). The charging voltage on the discharge capacitors is $6 \mathrm{kV}$. Breakdown occurs at $t$ $=2.5 \mu \mathrm{s}$. Note the merging parts of the prominences and how one prominence expands and evolves faster than the other.

region becomes extremely intense and localized (see Fig. 7). The counter-helicity signal for the unfiltered $\mathrm{x}$-ray diode is $\approx 23$ times larger than theco-helicity signal. This ratio is $\approx 20$ for the $\mathrm{x}$-ray diode with an $\mathrm{Al}$ filter and $\approx 50$ for the $\mathrm{x}$-ray diode with a Ti filter. This observation suggests development of a high energy x-ray source in the counter-helicity case.

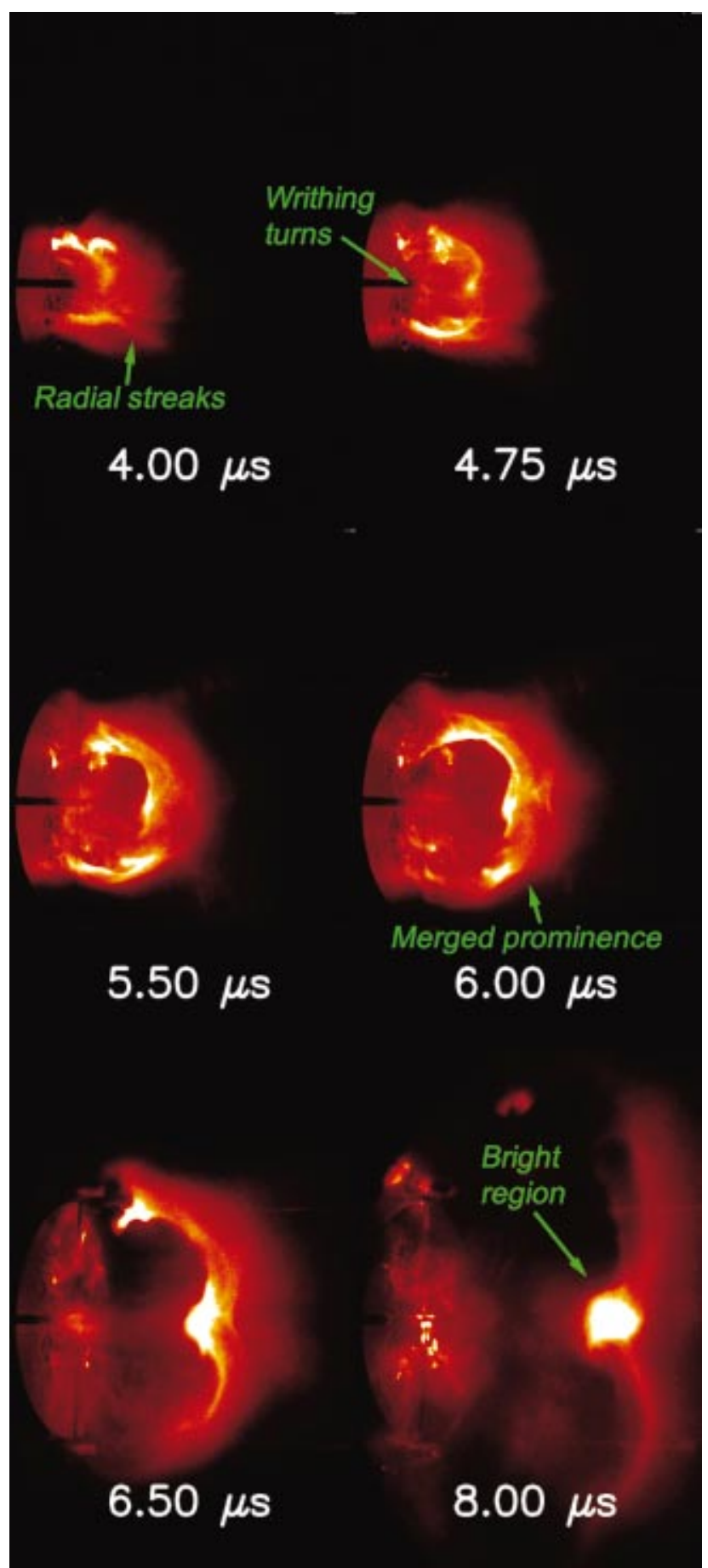

FIG. 4. (Color) Evolution of two prominences in the counter-helicity case (perpendicular view). Indicated times are measured from when the trigger pulse is applied to the discharge capacitor bank (at $t=0$ in Fig. 6). The charging voltage on the discharge capacitors is $6 \mathrm{kV}$. Breakdown occurs at $t=2.5 \mu \mathrm{s}$. Ejection of the plasma along radial streaks is seen at $4.0 \mu \mathrm{s}$ (compare with first frame of Fig. 3). Note the bright region between the prominences $(t \geqslant 6.5 \mu \mathrm{s})$.

\section{Comparison of co- and counter helicity expansion speed}

At a discharge capacitor charging voltage of $3 \mathrm{kV}$, the plasma expansion in the counter-helicity configuration is much faster than both the co-helicity and single prominence configuration (see Fig. 8 top). In contrast, at $6 \mathrm{kV}$ charging 




FIG. 5. (Color) In frame (a), two loops of plasma are seen at $6.5 \mu$ s after application of a $3 \mathrm{kV}$ discharge voltage on the electrodes in the counterhelicity configuration (perpendicular view). Frame (b) shows the perpendicular view of the plasma at a $6 \mathrm{kV}$ discharge voltage, in the same configuration at $5.0 \mu \mathrm{s}$. Writhing loops of plasma are visible at the higher discharge voltage in frame (b), which is consistent with annihilation of the toroidal magnetic field.

voltage, the plasma expansion speed is similar for all three configurations (see Fig. 8 bottom). The increase of plasma expansion speed in the $3 \mathrm{kV}$ counter-helicity configuration must be the result of a change in either the hoop-force, tending to increase expansion, or the toroidal field line tension, the only restoring force in this case (there is no strapping field). The observed increase of expansion speed at $3 \mathrm{kV}$ but not at $6 \mathrm{kV}$ is consistent with a local annihilation of toroidal magnetic field; the lack of difference between co- and counter helicity expansion speeds at $6 \mathrm{kV}$ is because the hoop force associated with the prominence current completely overwhelms the restoring force, so that the strength of the restoring force is not important (at $6 \mathrm{kV}$ charging voltage, the observed discharge current is $108 \mathrm{kA}$, much higher than the $43 \mathrm{kA}$ current at $3 \mathrm{kV}$ ). On a larger scale, north and south poles originally linked vertically in Fig. 1(b) will, after the annihilation process, be linked horizontally as well, i.e., significant magnetic fluxes will now go from both north poles to both south poles.

\section{E. Variation of mass influx boundary condition}

During operation with gas injected through two diagonally opposite electrodes only, it was observed that the prominence would, depending on which diagonal was chosen for gas injection, either remain in an S-shape much longer than in the original single prominence case [diagonal indicated in Fig. 1(d)], or it would skip the S-shape phase completely such that the plasma would be helical upon formation.

\section{F. Discussion}

Previous studies by other groups have examined the interaction between two merging spheromaks in co- and counter-helicity configurations ${ }^{17-19}$ and have shown that there is substantial conversion of magnetic energy into ion energy for the case of counter-helicity merging. These spheromak plasmas are fairly uniformly distributed across the cross section of the toroidal envelope, i.e., the current channel does not writhe about its axis. When two of these identical toroidal plasmas merge in co-helicity configuration (by having the toroidal currents and the toroidal fields in parallel), the magnetic field lines in the boundary region "meet" at an angle $2 \varphi$ (where $\varphi$ is the complement of the pitch angle of each spheromak) and the merging results in annihilation of the poloidal field. This means that a current sheet must form between the two spheromaks, and that any merging will be preceded by a reconnection of field lines at the boundary. For counter-helicity merging (parallel toroidal currents, antiparallel toroidal fields) the relative angle between merging fields is $\pi$ and the merging results in annihilation of the toroidal field. Since the toroidal field is typically stronger than the poloidal field (the poloidal field is due to the toroidal current), counter-helicity merging liberates much more energy than co-helicity merging.

A difference between these earlier merging spheromak studies and our merging prominence experiment is that each prominence in our experiment has a writhing current channel, i.e., the plasma is not uniformly distributed across the cross section of a toroidal envelope. Each current channel is nevertheless still governed by the force-free state equation, and is aligned with the magnetic field. Co-helicity merging is not particularly dramatic in our experiments judging from images, $\mathrm{x}$-ray emissions, and voltage traces, as compared to the counter-helicity merging. In the co-helicity camera images it appears that the leading parts of the prominences do merge. This type of merging has been indicated in numerical simulations. ${ }^{20}$

Before co-helicity merging, there already appears to be significant interaction between the two prominences and the nature of this interaction suggests why one prominence always expands faster than the other. In some images, a strand of plasma can be seen connecting one prominence with the other. This suggests that some fraction of the current which initially flowed through one prominence commutates to flow in the other. A larger toroidal current means a larger hoopforce and a faster expansion in the prominence receiving the transferred current. This is important for solar prominences because it indicates that one of two neighboring interacting prominences should erupt sooner than a solitary prominence would. Our experimental observation may predict which of the two prominences will erupt sooner; in an image with the toroidal currents flowing toward the top of the image, the prominence to the right erupts first if the prominences are right-handed, the one to the left if the prominences are lefthanded. The reason for this consistent dependence on orientation is not known.

Comparison of the co- and counter-helicity cases shows that the bias field polarity is critical to the plasma behavior-a simple reversal of the leads to a pair of coils dramatically changes the character and evolution of the plasma. In the counter-helicity case, the prominences have oppositely directed toroidal magnetic fields, requiring the formation of a much stronger current sheet in the region between the main current channels. It is clear from Table I that larger current densities correspond to greater x-ray production. Consequently, the extreme increase in $\mathrm{x}$ rays in the counter-helicity case is consistent with the existence of a much stronger current sheet. We surmise that electron- 

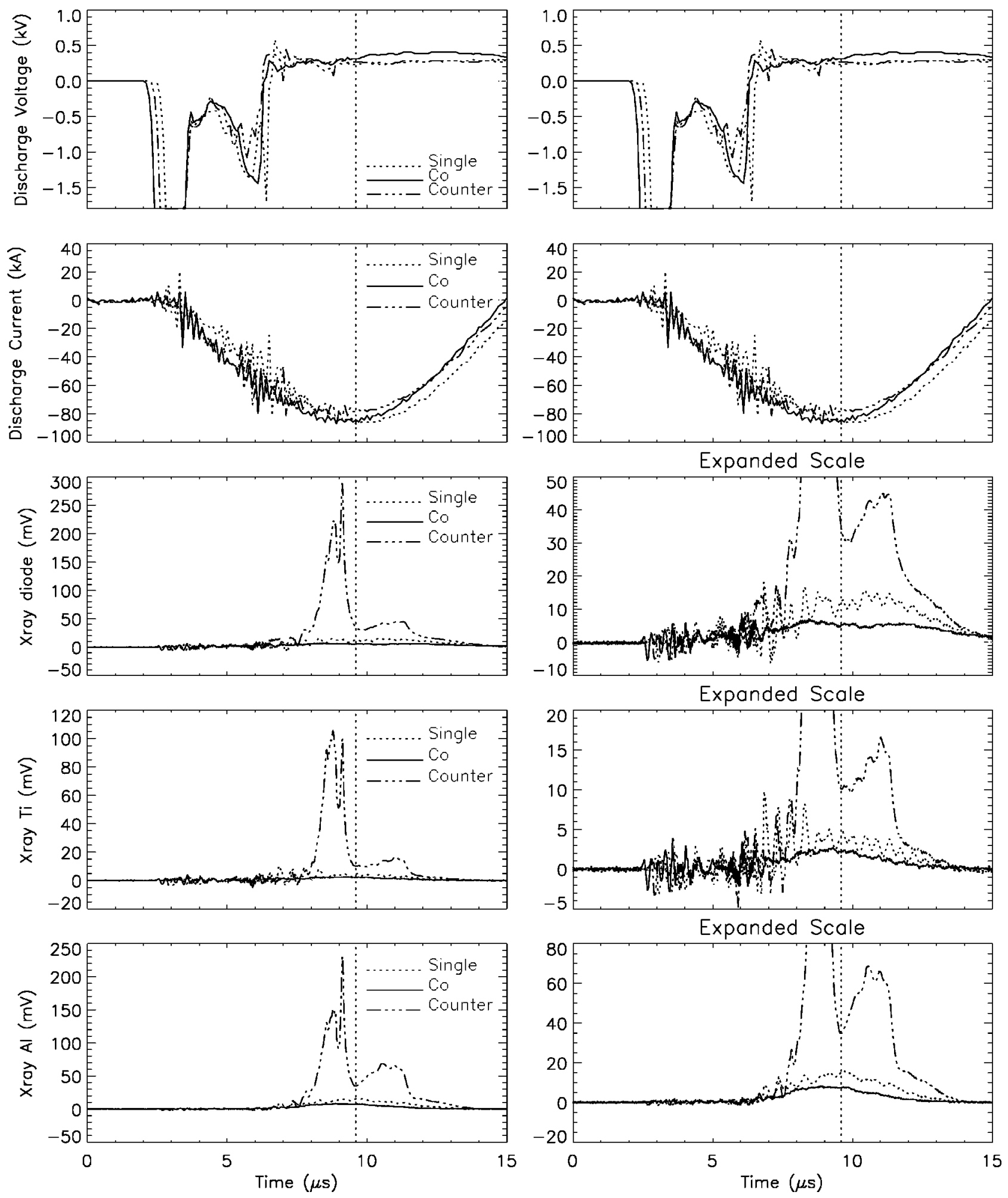

FIG. 6. Single prominence, co- and counter-helicity signals from the plasma are shown by dotted, solid, and dashed-dotted curves, respectively. These shots were taken at the discharge capacitor charging voltage of $6 \mathrm{kV}$. All other parameters were fixed. The $\mathrm{x}$-ray diode, $\mathrm{x}$-ray Ti, and $\mathrm{x}$-ray Al signals were taken from the $\mathrm{x}$-ray diode without any filter, with a Ti filter, and an $\mathrm{Al}$ filter, respectively. An order of magnitude higher $\mathrm{x}$-ray emission signal with a sharp rise is seen in the counter-helicity configuration. X-ray signals for the co-helicity and single prominence cases are clearly seen in the vertically expanded scales in the right set of the frames. The dotted vertical line in these frames coincides with the peak in the discharge current. Note that the $\mathrm{x}$-ray peaks in the counter-helicity configuration appear before the maximum in the discharge current.

neutral and ion-neutral collisions in this current sheet cause the bright region visible between the prominences in the camera images. The camera image would then constitute a direct observation of the reconnection layer.
The counter-helicity reconnection process results in the destruction of toroidal magnetic flux; this destruction is evident from counting the number of turns $N$ of the current channels around their respective toroidal envelope axes (i.e., 




FIG. 7. (Color) In this figure, the voltage dependence of the discharge is shown in the counter-helicity configuration (perpendicular views). The timing of these frames are chosen to show the final stage of the discharge, when the loops (as seen in Fig. 5) disappear and a bright region develops halfway between the foot-points. Except for the charging voltage on the discharge capacitor, all other parameters were fixed. Note that the bright region becomes more distinct and localized at higher discharge voltages.

in tokamak geometry, $N$ is the number of poloidal turns for half a toroidal turn). For times $t \gtrsim 4.5 \mu \mathrm{s}$, the camera images show $N \geqslant 6$, or three times as many turns as at comparable times for a solitary prominence. A larger $N$ corresponds to a larger ratio between the toroidal current, $I_{\mathrm{tor}}$, and the toroidal magnetic field, $B_{\text {tor }}$. Consequently, $B_{\text {tor }}$ must be much less in the counter-helicity case than in the single prominence case. (By virtue of operating with two initially identical prominences, $I_{\text {tor }}$ should be roughly one-half of what it is in a single prominence driven by the same power supply. In other words $B_{\text {tor }}$ must be much less than half of what it is for a single prominence.) Since the toroidal magnetic field provides the effective restoring force that resists prominence eruption, this means that each prominence in the counterhelicity case is more unstable than it would be if it were alone. This is important since it indicates that two interacting counter-helicity prominences on the Sun should erupt sooner than would be the case for similar noninteracting prominences. It has previously been suggested on the basis of geometry and inductance arguments, that only co-helicity reconnection is allowed on the Sun. ${ }^{21}$ Our observations show
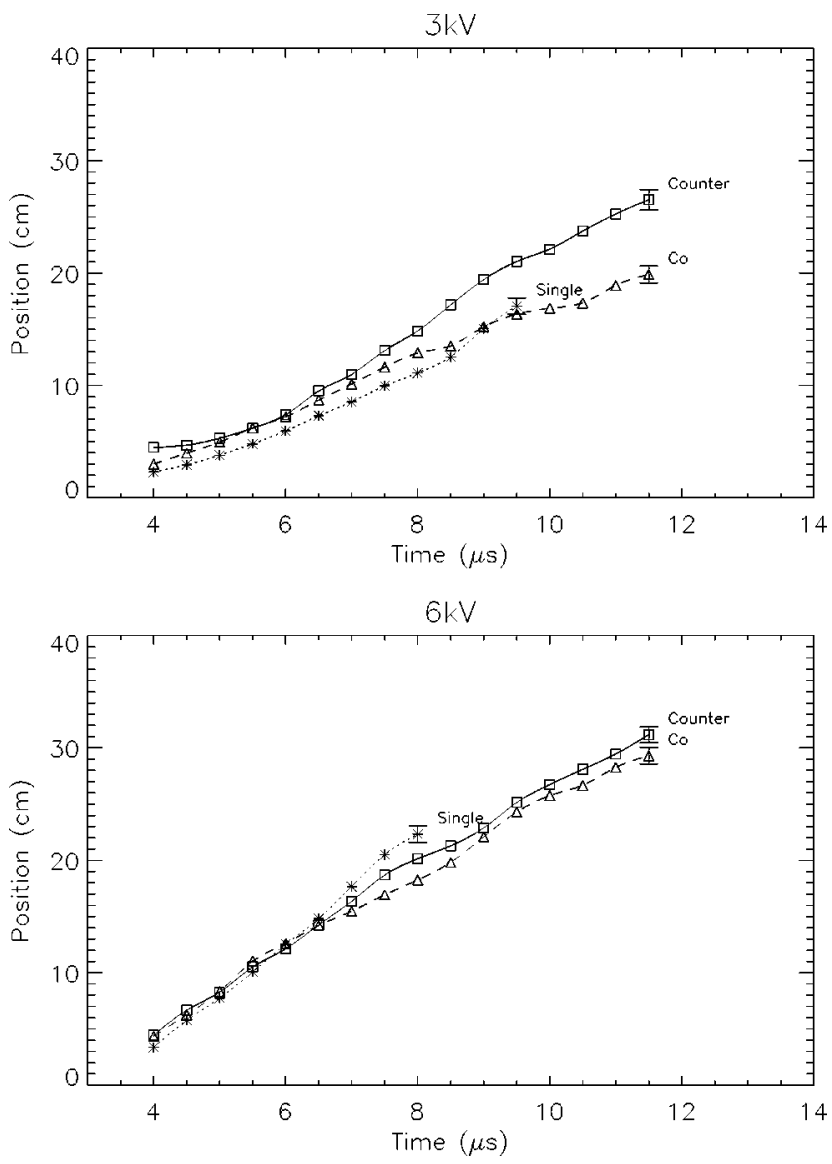

FIG. 8. Distance $h$ (defined in Fig. 2) of the evolving plasma vs time. These curves are plotted for single prominence, co-helicity, and counter-helicity configurations at $3 \mathrm{kV}$ and $6 \mathrm{kV}$ charging voltages. Time is measured from when the trigger pulse is applied to the discharge capacitor. Maximum error bars for each configuration are displayed at the end of the curves. At $3 \mathrm{kV}$, the plasma expands faster in the counter-helicity configuration than in the other two configurations, but at $6 \mathrm{kV}$ the plasma expands at similar rates for all three configurations.

to the contrary that counter-helicity reconnection in this geometry is not only possible but is very energetic and could be a candidate source for solar flares.

Bias field configuration (co- or counter-helicity) is not the only surface boundary condition affecting plasma behavior. The gas injection arrangement is also important and can be considered as a boundary condition on mass flux normal to the surface. By enabling or cutting off gas flow from selected electrodes, several different plasma regimes can be examined. For example, gas injection only through diagonally opposite electrodes produced a prominence with an orientation of the bias magnetic field at an angle with respect to the foot-points (i.e., with the neutral line at $45^{\circ}$ relative to the line between foot-points instead of at $90^{\circ}$ ). The prominence in this case evolved a more pronounced S-shape than in earlier experiments.

\section{SUMMARY AND CONCLUSIONS}

A four electrode magnetized plasma gun with individually controllable bias magnetic fields on each electrode and independent gas feeds at each electrode provides a great deal of flexibility for examining different prominence configura- 
tions. Reversing the polarity of the bias magnetic field for one prominence changes the merging from co- to counterhelicity and results in remarkably different behavior. The gas feed boundary condition also plays an important role in the experiment, as it enables single prominence experiments (by turning off the gas for two electrodes) including a single prominence at an angle to the neutral line. The results presented here, a semiquantitative survey of the plasmas produced by different source configurations, demonstrate the existence of several distinct regimes and also provide a preliminary indication of the essential dynamics governing these regimes. It is planned to examine these regimes in more detail in future experiments.

\section{ACKNOWLEDGMENTS}

The authors wish to thank F. C. Cosso, D. Felt, and L. Begay for their technical assistance.

Work supported by U.S. DOE Grant No. DE-FG0398ER54461. Much of the work described here is from the doctoral dissertation of one of the authors (J.F.H.).

${ }^{1}$ E. Tandberg-Hanssen, The Nature of Solar Prominences (Kluwer Academic, Dordrecht, 1995).
${ }^{2}$ J. F. Hansen and P. M Bellan, Astrophys. J. Lett. 563, L183 (2001).

${ }^{3}$ D. M. Rust and A. Kumar, Sol. Phys. 155, 69 (1994).

${ }^{4}$ J. Feynman and S. F. Martin, J. Geophys. Res., [Space Phys.] 100, 3355 (1995).

${ }^{5}$ A. A. Pevtsov, R. C. Canfield, and H. Zirin, Astrophys. J. 473, 533 (1996).

${ }^{6}$ A. M. Uralov, S. V. Lesovoi, V. G. Zandanov, and V. V. Grechnev, Sol. Phys. 208, 69 (2002).

${ }^{7}$ K. Tanaka, Sol. Phys. 136, 133 (1991).

${ }^{8}$ H. Zirin, Sol. Phys. 14, 328 (1970).

${ }^{9}$ M. Gigolashvili, Sol. Phys. 60, 293 (1978).

${ }^{10}$ S. F. Martin, W. H. Marquette, and R. Bilimoria, in The Solar Cycle, edited by K. L. Harvey, ASP Conf. Series 27, 53 (1992).

${ }^{11}$ A. A. Pevtsov, R. C. Canfield, and T. R. Metcalf, Astrophys. J. Lett. 440, L109 (1994).

${ }^{12}$ D. H. MacKay, A. W. Longbottom, and E. R. Priest, Sol. Phys. 185, 315 (1999).

${ }^{13}$ K. Kusano, T. Yokoyama, T. Maeshiro, and T. Sakurai, Adv. Space Res. 32, 1931 (2003).

${ }^{14}$ Z. Mikić, C. Barnes, and D. D. Schnack, Astrophys. J. 328, 830 (1988).

${ }^{15}$ Z. Mikić and J. A. Linker, Astrophys. J. 430, 898 (1994).

${ }^{16}$ P. M. Bellan and J. F. Hansen, Phys. Plasmas 5, 1991 (1998).

${ }^{17}$ M. Yamada, H. Ji, S. Hsu, T. Carter, R. Kulsrud, N. Bretz, F. Jobes, Y. Ono, and F. Perkins, Phys. Plasmas 4, 1936 (1997).

${ }^{18}$ Y. Ono, M. Inomoto, T. Okazaki, and Y. Udea, Phys. Plasmas 4, 1953 (1997).

${ }^{19}$ M. R. Brown, Phys. Plasmas 6, 1717 (1999).

${ }^{20}$ M. G. Linton, R. B. Dahlburg, and S. K. Antiochos, Astrophys. J. 553, 905 (2001)

${ }^{21}$ D. B. Melrose, Astrophys. J. 486, 521 (1997). 